\title{
Rac1 GTPase Inhibition Blocked Podocyte Injury and Glomerular Sclerosis during Hyperhomocysteinemia via Suppression of Nucleotide-Binding Oligomerization Domain- Like Receptor Containing Pyrin Domain 3 Inflammasome Activation
}

\author{
Qinghua Zhang Sabena M. Conley Guangbi Li Xinxu Yuan Pin-Lan Li \\ Departments of Pharmacology and Toxicology, Virginia Commonwealth University, \\ Richmond, VA, USA
}

\section{Keywords}

Rac1 GTPase · Membrane rafts · Inflammatory machinery · Glomerulosclerosis ·

Reactive oxygen species

\begin{abstract}
Elevated homocysteine (Hcy) levels have been shown to activate nucleotide-binding oligomerization domain-like receptor containing pyrin domain 3 (NLRP3) inflammasome leading to podocyte dysfunction and glomerular injury. However, it remains unclear how this inflammasome activation in podocytes is a therapeutic target for reversal of glomerular injury and ultimate sclerosis. The present study tested whether inhibition of Rac1 GTPase activity suppresses NLRP3 inflammation activation and thereby blocks podocyte injury induced by elevated Hcy. In cultured podocytes, we found that L-Hcy (the active Hcy form) stimulated the NLRP3 inflammasome formation, as shown by increased colocalization of NLRP3 with apoptosis-associated speck-like protein (ASC) or caspase-1, which was accompanied by increased interleukin$1 \beta$ production and caspase-1 activity, indicating NLRP3 inflammasome activation. Rac1 activator, uridine triphosphate (UTP), mimicked L-Hcy-induced NLRP3 inflammasome activation, while Rac1 inhibitor NSC23766 blocked it. This Rac1 inhibition also prevented L-Hcy-induced podocyte dysfunction. All these effects were shown to be mediated via lipid raft redox signaling platforms with nicotinamide adenine dinucleotide phosphate oxidase subunits and consequent $\mathrm{O}_{2}^{-}$production. In animal studies, hyperhomocysteinemia ( $\mathrm{hHcy}$ ) induced by folate-free diet was shown to induce NLRP3 inflammasome formation and activation in glomeruli, which
\end{abstract}




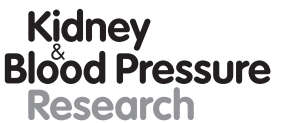

Research

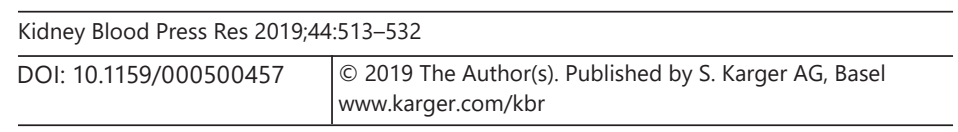

Zhang et al.: NLRP3 Inflammasome Activation via LR Redox Signaling Platforms

was also mimicked by UTP and inhibited by NSC23766 to a comparable level seen in Nlrp3 gene knockout mice. These results together suggest that Rac1 inhibition protects the kidney from $\mathrm{hHcy}$-induced podocyte injury and glomerular sclerosis due to its action to suppress NLRP3 inflammasome activation in podocytes.

\section{Introduction}

Hyperhomocysteinemia (hHcy) is considered as a risk factor for cardiovascular diseases such as atherosclerosis and hypertension. Recent studies in our laboratory and by others have demonstrated that hHcy is one of the important pathogenic factors to result in the progression of glomerular injury into end-stage renal disease [1-5]. However, the precise mechanism mediating hHcy-induced glomerular sclerosis is still not very clearly clarified. Recently, the activation of nucleotide-binding oligomerization domain-like receptor containing pyrin domain 3 (NLRP3)-centered inflammasome has been shown to contribute to the onset or gradual progression of glomerular injury under different pathological conditions. This type of inflammasome has been well characterized as an intracellular machinery that turns on the inflammatory response and instigates damage in the glomeruli, ultimately leading to glomerular sclerosis [6-8]. In the kidney, inhibition of this inflammasome activation has been considered as an innovative therapeutic strategy to possibly prevent or treat glomerular diseases associated with local inflammation [6-9]. Our previous studies have shown that the activation of this NLRP3 inflammasome is a critical molecular mechanism mediating homocysteine (Hcy)-induced podocyte injury and ultimately leading to glomerular sclerosis [9]. However, it remains unknown whether this Hcy-activated NLRP3 inflammasome can be a therapeutic target for prevention or treatment for progression of glomerular sclerosis.

There is accumulating evidence that the pathogenic action of hHcy is dependent on the formation and activation of membrane lipid raft (LR) redox signaling platforms associated with nicotinamide adenine dinucleotide phosphate oxidase (NOX). It is well known that NOX is composed of membrane-bound subunits gp $91^{\text {phox }}$ and $\mathrm{p} 22^{\text {phox }}$ as well as cytosolic subunits $\mathrm{p} 47^{\text {phox }}$, p40 ${ }^{\text {phox }}$, and $\mathrm{p} 67^{\text {phox }}[10]$. Upon stimulation, LRs as membrane lipid microdomains can be clustered to form signaling platforms, which drive NOX subunits aggregation and assemble as an enzyme complex to mediate redox signaling and thereby regulate a variety of cellular activity and cell function $[11,12]$. This redox signaling platform can be activated by Hcy and other pathological stimuli and thereby generates superoxide $\left(\mathrm{O}_{2}{ }^{-}\right)$, leading to glomerular local oxidative stress and inflammation. It has been suggested that NOX-driven glomerular redox regulation is implicated in the pathogenesis of various glomerular diseases including diabetic nephropathy, hHcy-induced nephropathy, and hypertension or obesity-related chronic glomerular diseases [13]. As one of triggering mechanisms, this NOX-mediated reactive oxygen species (ROS) have also been reported to activate NLRP3 inflammasomes in podocytes during hHcy. In this regard, both $\mathrm{O}_{2}{ }^{-}$and $\mathrm{H}_{2} \mathrm{O}_{2}$ have been found to instigate NLRP3 inflammasome formation and activation by changing thioredoxin binding to NLRP3 domains [14, 15]. Further studies have reported that NOX activation to produce $\mathrm{O}_{2}{ }^{-}$is dependent upon Vav2-mediated Rac1 GTPase activity [13]. It is now imperative to test whether Rac1 inhibition may reduce NLRP3 inflammasome formation and activation in podocytes and thereby protect the kidney from podocyte injury and glomerular sclerosis. 


\section{Kidney \\ Blood Pressure \\ Research}

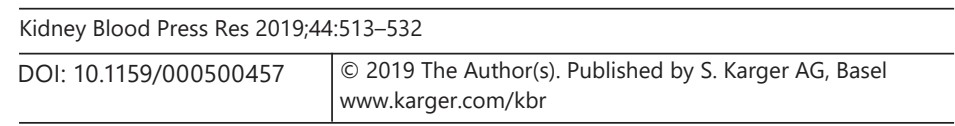

Zhang et al.: NLRP3 Inflammasome Activation via LR Redox Signaling Platforms

In the present study, we tested the potential that Rac1 inhibition suppresses NLRP3 activation in podocytes during hHcy and thereby prevents podocyte injury and glomerular sclerosis. We first performed cell studies in vitro to determine whether Rac1 is involved in NLRP3 activation by Hcy through LR redox signaling platforms on podocyte membrane. Then, we conducted animal studies to confirm the therapeutic role of pharmacological intervention of Rac1 activity in protecting or enhancing hHcy-induced podocyte injury and glomerular sclerosis. Our results indicate that NLRP3 inflammasome activation may serve as a therapeutic target for potential to prevent or reverse podocyte dysfunction and glomerular and sclerosis during hHcy.

\section{Materials and Methods}

\section{Cell Culture}

A conditionally immortalized mouse podocyte cell line (a kind gift from Dr. Paul E. Klotman, Mount Sinai School of Medicine) was cultured undifferentiated with $10 \mathrm{U} / \mathrm{mL}$ recombinant mouse interferon- $\gamma$ at $33^{\circ} \mathrm{C}$ on collagen I-coated flasks in RPMI 1640 medium containing $10 \%$ fetal bovine serum, $100 \mathrm{U} / \mathrm{mL}$ penicillin, and $100 \mathrm{mg} / \mathrm{mL}$ streptomycin. Prior to experiments, podocytes were allowed to differentiate at $37^{\circ} \mathrm{C}$ for $10-14$ days in the absence of interferon- $\gamma$ and afterward used for experiments. Cells were treated with L-Hcy ( $40 \mu \mathrm{M})$ for $24 \mathrm{~h}$, which was shown to be the optimal concentration and time to produce a significant activation of the NLRP3 inflammasome in our previous studies [14]. Pharmacological interventions of uridine triphosphate (UTP; $100 \mu \mathrm{M})$ and NSC-23766 $(50 \mu \mathrm{M})$ were added to the cells $1 \mathrm{~h}$ prior to L-Hcy treatment.

\section{Animals}

To produce a model of hHcy, 8-weeks-old C57BL/6J wild-type mice and Nlrp3 knockout (Nlrp3 KO, Mutant Mouse Research and Resource Center) mice were uninephrectomized and allowed 1 week for surgery recovery. Nlrp3 $\mathrm{KO}\left(\mathrm{Nlrp3}^{-/-}\right)$and wild-type $\left(\mathrm{Nlrp}^{{ }^{+/+}}\right)$mice were genotyped using PCR. Detection of a PCR product at $254 \mathrm{bp}$ indicates Nlrp3 ${ }^{-/-}$, while a PCR product of $330 \mathrm{bp}$ indicates Nlrp3 $3^{+/+}$mice, which were described in detail in our previous studies [5]. Uninephrectomized mice were fed either a normal diet (ND) or a folate-free (FF) diet to induce hHcy (Dyets Inc., Bethlehem, PA, USA) for 6 weeks. Uninephrectomization was used to speed up glomerular injury induced by hHcy. Groups of mice received intraperitoneal injections of the Rac1 activator, UTP, or Rac1 inhibitor, NSC-23766 (1 mg/kg/day), throughout the ND or FF diet feeding period, and doses of these compounds were chosen according to recent reports [16]. When experimental treatments are completed, a 24-h urine sample was collected from each mouse using small rodent metabolic cages. Then, mice were sacrificed and their kidneys were harvested for biochemical and molecular analyses. All animal studies and related protocols were approved by the Institutional Animal Care and Use of the Committee of Virginia Commonwealth University.

\section{HPLC Quantitation of Plasma Hcy Concentrations}

Plasma total Hcy levels were measured as we described previously [17]. In brief, $100 \mu \mathrm{L}$ plasma or standard solution was mixed with $10 \mu \mathrm{L}$ of internal standard, thionglycolic acid (2.0 $\mathrm{mmol} / \mathrm{L}$ ), and then treated with $10 \mu \mathrm{L}$ of $10 \%$ tri-n-butylphosphine solution in dimethylformamide at $4{ }^{\circ} \mathrm{C}$ for $30 \mathrm{~min}$. Then, $80 \mu \mathrm{L}$ of ice-cold $10 \%$ trichloroacetic acid in $1 \mathrm{mmol} / \mathrm{L}$ EDTA was added and centrifuged to remove proteins in the sample. A total of $100 \mu \mathrm{L}$ of the supernatant was transferred into the mixture of $20 \mu \mathrm{L}$ of $1.55 \mathrm{M}$ sodium hydroxide, $250 \mu \mathrm{L}$ of 0.125 $\mathrm{M}$ borate buffer ( $\mathrm{pH}$ 9.5), and $100 \mu \mathrm{L}$ of $1.0 \mathrm{mg} / \mathrm{mL}$ ABD-F solution. The resulting mixture was 


\section{Kidney \\ Blood Pressure \\ Research}

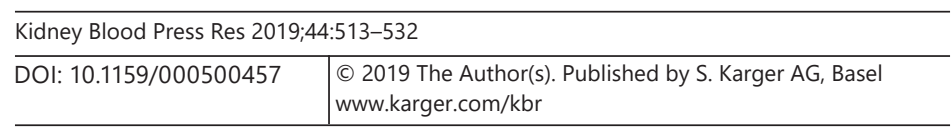

Zhang et al.: NLRP3 Inflammasome Activation via LR Redox Signaling Platforms

incubated at $60^{\circ} \mathrm{C}$ for $30 \mathrm{~min}$ to accomplish derivatization of thiols. HPLC was performed, and separation was carried out at an ambient temperature on an analytical column, Supelco LC-18-DB (1,504.6 mm ID, $5 \mathrm{~m}$ ) with a Supercoil LC-18 guard column (204.6 mm ID, $5 \mathrm{~m})$.

\section{Confocal Microscopy and Immunofluorescent Staining of Frozen Tissues and Cells}

To observe colocalization of inflammasome components and podocyte marker proteins, indirect immunofluorescent staining was used in frozen tissue sections and cultured podocytes. Podocytes seeded in 8-well chambers were fixed in 4\% paraformaldehyde (PFA), washed with phosphate-buffered saline (PBS), and blocked with $1 \%$ bovine serum albumin in PBS before being incubated in primary antibodies $(1: 100)$ overnight at $4{ }^{\circ} \mathrm{C}$. Then, the primary antibodies against NLRP3 inflammasome components and podocyte protein markers were used for the detection of their expression and interactions as inflammasome complexes. These antibodies include goat anti-NLRP3 (Abcam, Cambridge, MA, USA), rabbit anti-apoptosis-associated specklike protein (ASC; Santa Cruz, Santa Cruz, CA, USA), or rabbit anti-caspase-1 (Santa Cruz) antibodies. Frozen slides with mouse kidney tissue were fixed in pre-cold acetone, blocked with 3\% donkey serum, then incubated with the same primary antibodies (1:50) mentioned above overnight at $4{ }^{\circ} \mathrm{C}$. To detect kidney podocyte injuries, additional groups of podocytes and frozen kidney sections were only stained using antibodies against podocyte markers, podocin (1:50; Sigma, St. Louis, MO, USA) or desmin (1:50; BD Biosciences, San Jose, CA, USA) or Vav2 as a marker of Rac1 activation (Santa Cruz, Santa Cruz, CA, USA). Double immunofluorescent staining for confocal microscopy was performed by Alexa-488- or Alexa-555-labeled secondary antibodies (1:200 podocytes, 1:100 frozen kidney slides; Life Technologies, Grand Island, NY, USA) with an incubation of $1 \mathrm{~h}$ at room temperature, which detects the colocalization of NLRP3 inflammasome components, which indicates NLRP33 inflammasome formation. Slides were then washed, mounted, and observed using a confocal laser scanning microscope (Fluoview FV1000, Olympus, Japan), and tools in Image Pro Plus 6.0 software (Media Cybernetics, Bethesda, MD, USA) were used to analyze colocalization or the degree of overlap between the 2 wavelengths, which was expressed as the Pearson correlation coefficient.

\section{Measurement of LR Clusters with NOX Subunits in Podocytes}

For confocal microscopic detection of LRs and their associated proteins, podocytes grown on poly-L-lysine-coated chambers were treated with compounds mentioned above. Then, podocytes were washed with cold PBS, fixed for 15 min with 4\% PFA and then blocked with 1\% bovine serum albumin in PBS for 30 min. GM1 gangliosides enriched in LRs were stained with Alexa488-labeled cholera toxin (CTX; 1 g/mL, Molecular Probes, Carlsbad, CA, USA) for $30 \mathrm{~min}$. The patch formation of Alexa488-labeled CTX and gangliosides complex represented the clusters of LRs. Clustering was defined as 1 or several intense spots of fluorescence on the cell surface, whereas unstimulated cells displayed a homogenous distribution of fluorescence throughout the membrane. To detect the colocalization of LRs and NOX subunits anti-gp91 ${ }^{\text {phox }}$ and anti-p4 $7^{\text {phox }}$, podocytes were incubated overnight with indicated goat or rabbit antibodies 1 1:100, BD bioscience), followed by incubation with $5 \mu \mathrm{g} / \mathrm{mL}$ Texas Red-conjugated anti-goat or anti-goat $\mathrm{II}^{\mathrm{O}}$ antibody for an additional $1 \mathrm{~h}$ at room temperature. After mounting, the slides were observed under confocal laser scanning microscope. In each experiment, the results were presented as the percentage of cells showing a cluster after the indicated treatment described above.

\section{Direct Fluorescent Staining of F-Actin}

To determine the role of Rac1 activity in L-Hcy-induced cytoskeleton changes in podocytes, these cells were seeded in 8-well chambers and treated with $40 \mu \mathrm{M} \mathrm{L}$-Hcy for $24 \mathrm{~h}$. After washing cells with PBS and 4\% PFA fixed cells for 15 min at room temperature, podocytes were permeabilized with $0.1 \%$ Triton X-100 and blocked with $3 \%$ bovine serum albumin. 


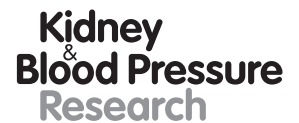

Kidney
Blood Pressure

Research

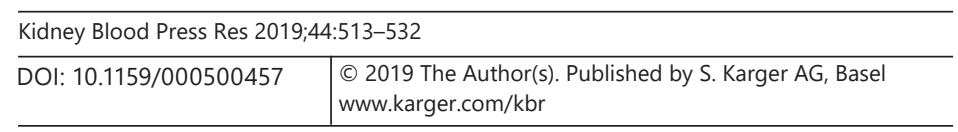

Zhang et al.: NLRP3 Inflammasome Activation via LR Redox Signaling Platforms

F-actin was stained with rhodamine-phalloidin (Invitrogen, Carlsbad, CA, USA) for 15 min at room temperature. Cells were examined by fluorescent microscopy after mounting. Cells with distinct F-actin fibers were counted as we described previously [18]. Scoring was obtained from 100 cells in each group.

\section{Immunohistochemistry}

Kidneys were perfused in 4\% PFA and removed and fixed in 10\% neutral buffered formalin. The 5- $\mu \mathrm{m}$ tissue sections embedded in paraffin were cut and mounted onto microscope slides. Deparaffination was induced through heat, tissue cleaning agent, ethanol, and water. Slides were boiled in $0.01 \mathrm{M}$ citrate buffer $\mathrm{pH} 6.0$ for $20 \mathrm{~min}$ to unmask antigen-binding sites. Endogenous peroxidase activity was blocked by incubating slides in 3\% hydrogen peroxide $\left(\mathrm{H}_{2} \mathrm{O}_{2}\right)$ in $100 \%$ methanol $(\mathrm{MeOH})$ for $30 \mathrm{~min}$. The sections were then incubated at room temperature for $30 \mathrm{~min}$ in $10 \%$ goat serum to block nonspecific binding and incubated

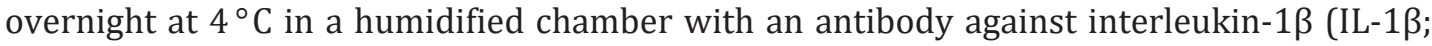
Abcam, Cambridge, MA, USA) diluted 1:50 in Tris-buffered saline with $0.2 \%$ Tween containing $4 \%$ goat serum. Then, the slides were incubated for $30 \mathrm{~min}$ at room temperature in a humidified chamber with a biotinylated goat anti-rabbit IgG-B antibody diluted 1:200 in Trisbuffered saline with $0.2 \%$ Tween. These slides were subsequently placed in streptavidinhorseradish peroxidase for $30 \mathrm{~min}$ at room temperature in a humidified chamber, then incubated with $50 \mu \mathrm{L}$ of diaminobenzadine (BioGenex, San Ramon, CA, USA) as a substrate, counterstained with hematoxylin (Sigma-Aldrich, Saint Louis, MO, USA), dehydrated, and fixed with Permount histological mounting medium (Fisher Scientific, Hampton, NH, USA).

\section{Assays of Caspase-1 Activity, IL-1 $\beta$ Production, and Vascular Endothelial Growth Factor}

Caspase- 1 activity (Biovision, Mountain View, CA, USA) was measured by a commercially available colorimetric assay. IL-1 $\beta$ production (R\&D Systems, Minneapolis, MN, USA) and vascular endothelial growth factor (VEGF; Bender Medsystems, San Diego, CA, USA) were quantified through an ELISA using cellular supernatant. All these assays were performed according to manufacturer's instructions.

\section{Electron Spin Resonance Spectrophotometry of $\mathrm{O}_{2}^{-}$Production}

Cellular protein samples were prepared by using modified Kreb's-Hepes buffer containing deferoximine $(100 \mu \mathrm{M})$ and diethyldithio-carbamate $(5 \mu \mathrm{M})$. NOX-dependent $\mathrm{O}_{2}{ }^{-}$production was examined by the addition of $1 \mathrm{~mm}$ nicotinamide adenine dinucleotide phosphate as a substrate in $30 \mu \mathrm{g}$ protein in the presence or absence of superoxide dismutase (SOD; $800 \mathrm{U} /$ $\mathrm{mL}$ ) and then supplied with $10 \mathrm{mM} \mathrm{O}_{2}{ }^{-}$specific spin trapping compound, 1-hydroxy3-methoxycarbonyl-2,2,5,5-tetramethylpyrrolidine $(\mathrm{CMH})$. The mixture was loaded in glass capillaries and immediately analyzed for $\mathrm{O}_{2}{ }^{-}$production kinetically for $10 \mathrm{~min}$ in an electron spin resonance (ESR) spectrometer. Results were obtained by subtracting the total 1-hydroxy3-methoxycarbonyl-2,2,5,5-tetramethylpyrrolidine signal without SOD, representing the SOD-specific signal. All values were expressed as the fold change from control.

\section{Morphological Examination}

Glomerular structure was examined using fixed paraffin-embedded kidneys, stained with a periodic acid-Schiff stain. Glomeruli were scored on a scale of 0-4 depending on the extent of sclerotic changes. In general, 50 glomeruli were counted under the microscope, 0 represents no lesion, 1 represents sclerosis $<25 \%$ of the glomerulus, while 2,3 , and 4 represent sclerosis of 25-50, $>50-75$, and $>75 \%$ of the glomerulus, respectively. The glomerular damage index (GDI) was composed of the average of these scores and was calculated according to the following formula: $([\mathrm{N} 1 \times 1]+[\mathrm{N} 2 \times 2]+[\mathrm{N} 3 \times 3]+[\mathrm{N} 4 \times 4]) / n$, where $\mathrm{N} 1, \mathrm{~N} 2, \mathrm{~N} 3$, and $\mathrm{N} 4$, 


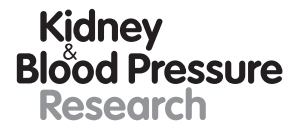

Kidney
Blood Pressure
Research

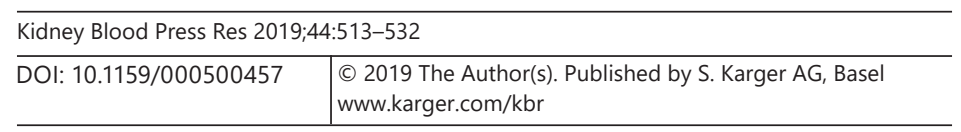

Zhang et al.: NLRP3 Inflammasome Activation via LR Redox Signaling Platforms

respectively, represent the number of glomeruli with scores of $1,2,3$, and 4, and $n$ represents the total number of scored glomeruli $[19,20]$. This observation was examined by 2 investigators and averaged under blind conditions.

\section{Assay of Rac1 GTPase Activity}

GTPase-linked immunosorbent assay was performed to determine Rac1 activation in vitro (Cytoskeleton, Denver, CO, USA). Podocytes were lysed in lysis buffer to prepare lysates by centrifugation at $10,000 \mathrm{~g}$ for $1 \mathrm{~min}$ at $4{ }^{\circ} \mathrm{C}$. Equalized amounts of protein $(1 \mathrm{mg} / \mathrm{mL})$ were loaded onto a Rac1 GTP affinity plate for $30 \mathrm{~min}$. Following incubation, the plate was washed in wash buffer and then incubated with primary and secondary antibodies against Rac1. The activated Rac1 was determined after exposure to HRP detection reagents by measuring absorbance at $490 \mathrm{~nm}$ using a microplate spectrophotometer.

\section{Statistical Analysis}

All data are presented as mean \pm standard error (SE); $n$ represents the number of independent experiments unless otherwise stated. Differences in mean values within and between multiple groups were analyzed using one-way analysis of variance followed by Dunnett's post hoc test. The statistically significant differences between 2 groups were assessed by applying the paired or unpaired Student $t$ test. The significance level was $p<0.05$.

\section{Results}

Effects of Rac1 Activity Inhibition and Enhancement on L-Hcy-Induced NLRP3

Inflammasome Formation in Podocytes

To determine whether Rac1 activity is critical for the NLRP3 inflammasome formation and activation, podocytes were treated with either UTP, a Rac1 activity enhancer through activation of its partner, Vav2, or NSC23766, a selective inhibitor of Rac1 activity in the presence or absence of L-Hcy. As shown in Figure 1a, pretreatment of podocytes with L-Hcy resulted in a significant elevation of GTP-bound Rac compared to vehicle-treated podocytes, indicating increases in Rac1 activity. UTP itself increased GTP-bound Rac, which was similar to the level of L-Hcy-induced enhancement of Rac1 activity. However, inhibition of Rac1 activity by NSC23766 treatment was observed, and L-Hcy-induced increase in Rac1 activity was also blocked by NSC23766.

By confocal microscopic analysis, we found increased colocalization of NLRP3 with ASC or caspase-1 in L-Hcy-treated podocytes when compared with vehicle-treated podocytes (Fig. 1b, c). Increase in Rac1 activity by UTP mimicked the L-Hcy-induced NLRP3 inflammasome formation, but it had no further enhancement on L-Hcy-induced effects on this inflammation formation in podocytes. In contrast, L-Hcy-induced increase in colocalization of NLRP3 inflammasome components, namely, the formation of NLRP3 inflammasome was blocked by Rac1 inhibitor, NSC23766. Summarized data of colocalization coefficient analysis are presented in Figure 1d and e, indicating that increases in Rac1 activity either by L-Hcy or by UTP enhance NLRP3 inflammasome formation in podocytes, while inhibition of Rac1 activity by NSC23766 suppresses L-Hcy-induced NLRP3 formation.

\section{Effects of Rac1 Activity Inhibition and Enhancement on L-Hcy-Induced NLRP3}

Inflammasome Activation in Podocytes

The activity of caspase- 1 in podocyte lysates and the level of secreted IL- $1 \beta$ in supernatant of podocyte cultures were measured to examine the effects of Rac1 activity inhibition and enhancement on L-Hcy-induced NLRP3 inflammasome activation. As shown in Figure 2a 


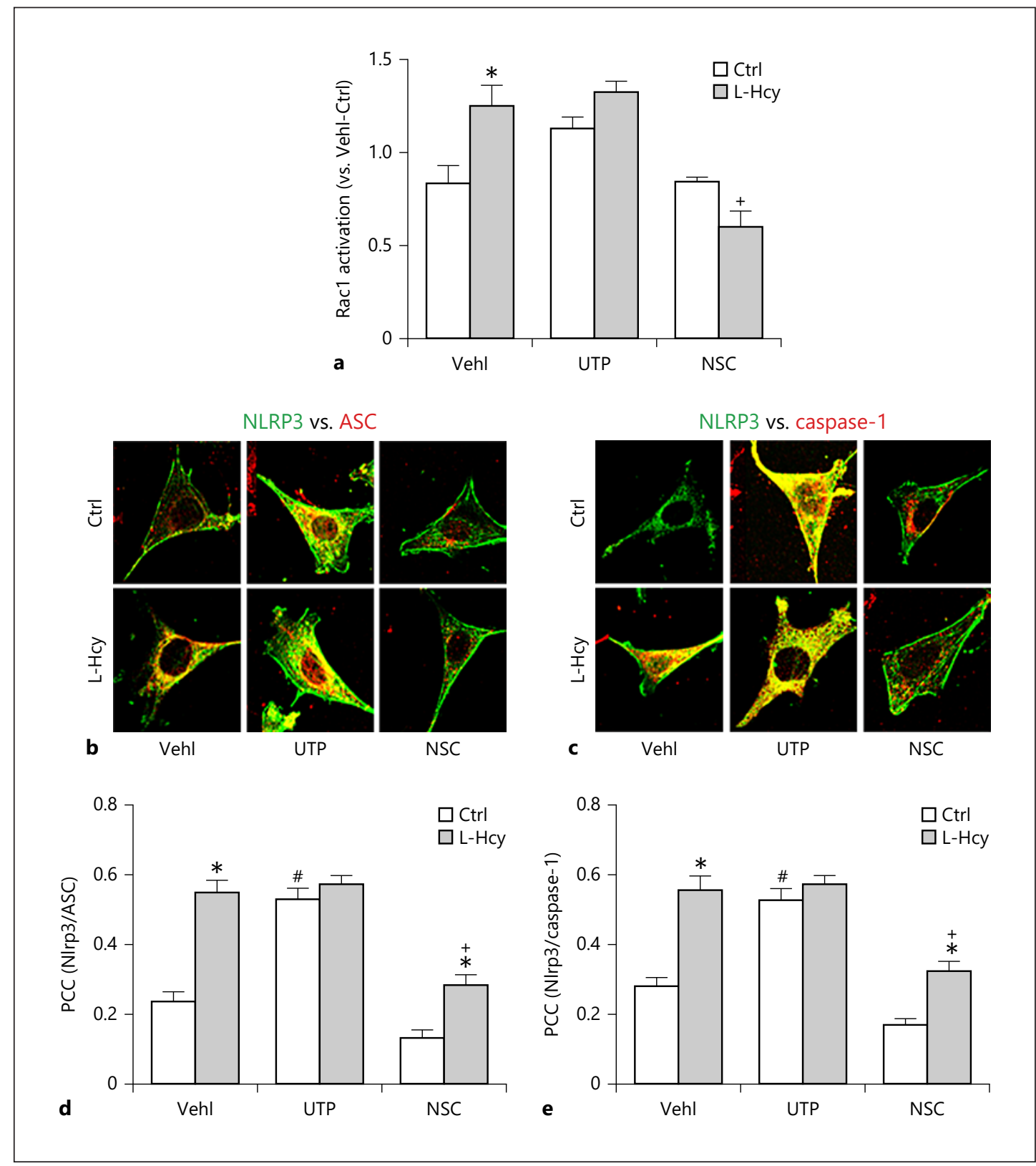

Fig. 1. Rac1 Inhibition prevented L-Hcy-induced NLRP3 inflammasome formation in podocytes. a Rac1 activity in UTP- and NSC23766-treated podocytes $(n=3)$. b and $\mathbf{c}$ Confocal images showing the colocalization of NLRP3 (green) with ASC (red; b) and NLRP3 (green) with caspase-1 (red; c) in cultured podocytes treated with UTP or NSC. $\mathbf{d}$ and e Summarized data showing the fold change in correlation coefficient $(n=6){ }^{*} p<0.05$ versus Ctrl; ${ }^{\#} p<0.05$ versus Vehl/Ctrl; ${ }^{+} p<0.05$ versus Vehl/L-Hcy. Vehl, vehicle; UTP, uridine triphosphate; NSC, NSC-23766; Hcy, homocysteine; NLRP3, NOD-like receptor containing pyrin domain 3; Ctrl, control.

and $b$, we found that L-Hcy increased caspase- 1 activity and IL- $1 \beta$ production in podocytes, which were similar to UTP-induced enhancement of caspase- 1 activity and IL- $1 \beta$ production. Treatment of podocytes by NSC23766 attenuated caspase- 1 activity and IL-1 $\beta$ production and completely blocked L-Hcy-induced effects on NLRP3 inflammasome activation as shown by no changes in caspase- 1 activity and IL- $1 \beta$ production upon L-Hcy stimulation. 
Fig. 2. Rac1 inhibition attenuated L-Hcy-induced NLRP3 inflammasome activation in podocytes. a Caspase-1 activity, shown as fold versus Vehl, measured in podocytes treated with Hcy, UTP, and NSC $(n=6)$. b IL-1 $\beta$ production measured in the supernatant of podocytes treated with Hcy, UTP, and NSC ( $n=7)$. c Measurement of VEGF secretion as a podocyte function parameter was detected in the supernatant of treated podocytes $(n=6) .{ }^{*} p<0.05$ versus Ctrl; ${ }^{\#} p<0.05$ versus Vehl/Ctrl; ${ }^{+} p<0.05$ versus Vehl/ L-Hcy. Vehl, Vehicle; UTP, uridine triphosphate; NSC, NSC-23766; Hcy, homocysteine; VEGF, vascular endothelial growth factor; IL$1 \beta$, interleukin- $1 \beta$.
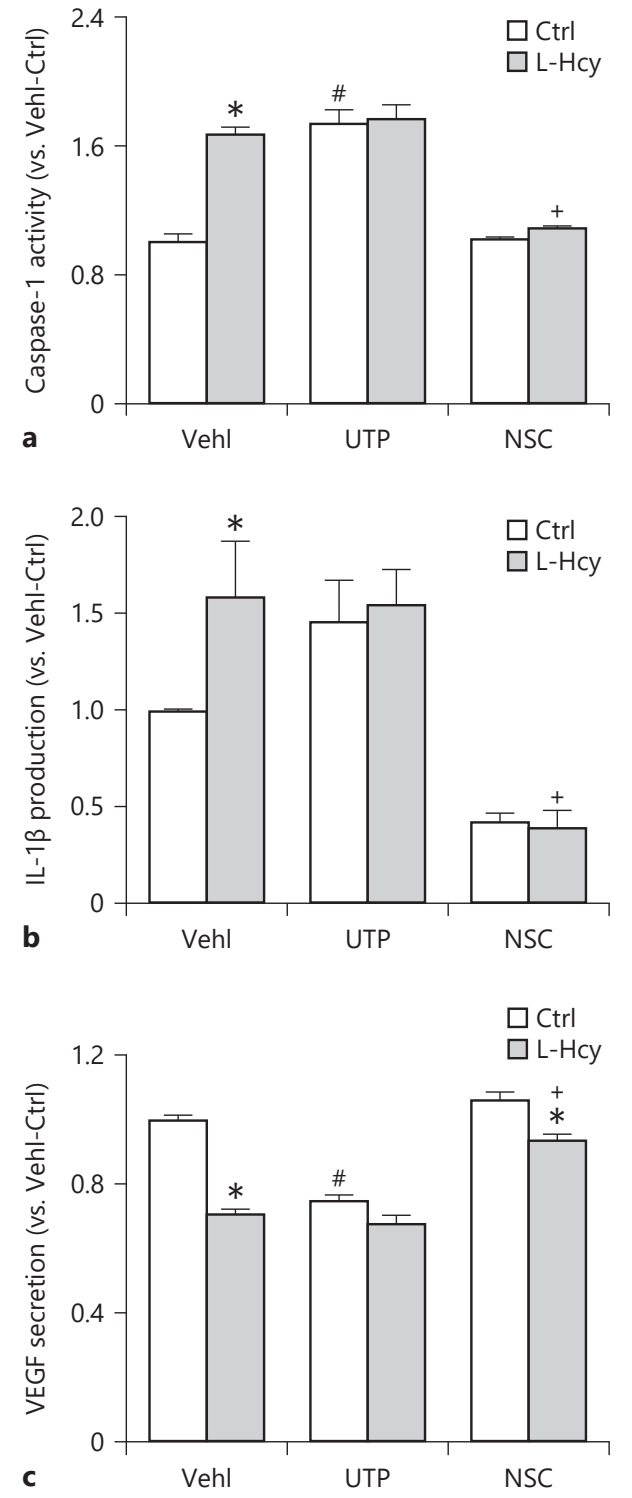

\section{Effects of Rac1 Activity Inhibition and Enhancement on L-Hcy-Induced Podocyte Dysfunction or Injury}

To determine the role of Rac1 activity in the control of podocyte function in the presence or absence of L-Hcy, we analyzed several parameters that represent podocyte function or injury markers. First, we detected the VEGF levels, a specific marker for mature and active podocytes. It was found that both L-Hcy and UTP significantly reduced VEGF secretion, while NSC23766 slightly increased the secretion of VEGF. In the presence of NSC23766, L-Hcyinduced inhibition of VEGF production was completely blocked (Fig. 2c). As shown in Figure 3 , the expression of podocytes injury marker, desmin, markedly increased, while podocin levels decreased upon L-Hcy stimulation or UTP treatment compared to vehicle-treated cells. Pretreatment of podocytes with NSC decreased L-Hcy-induced desmin increase and podocin decrease. Densitometric analysis showed that the expression of podocyte injury marker, 




Fig. 3. Attenuation of L-Hcy-induced podocyte dysfunction by Rac1 inhibition. $\mathbf{a}$ and $\mathbf{b}$ Immunofluorescence staining showed that activation of Vav2 by UTP or Rac1 inhibition by NSC rescued Hcy-induced expression of podocyte marker podocin as well as suppressed expression of podocyte injury marker desmin $(n=6)$. c and $\mathbf{d}$ Summarized graph showed the statistical data for podocin and desmin. e and $\mathbf{f}$ Microscopic images of F-actin proceeded rhodamine-phalloidin staining in podocytes and summarized data of distinct, longitudinal F-actin fibers. Scoring was from 100 podocytes in different groups $(n=6)$. ${ }^{*} p<0.05$ versus Ctrl; ${ }^{\#} p<$ 0.05 versus Vehl/Ctrl; ${ }^{+} p<0.05$ versus Vehl/L-Hcy. Vehl, vehicle; UTP, uridine triphosphate; NSC, NSC23766; Hcy, homocysteine.

desmin, significantly increased, but podocyte functional marker, podocin, significantly decreased when these cells were treated by L-Hcy or UTP. In the presence of NSC23766, the effects of L-Hcy to change podocyte marker level were almost completely blocked (Fig. 3c, d). Furthermore, stained F-actin with rhodamine-phalloidin in vehicle-treated podocytes exhibited well-defined F-actin fibers, which run along the longitudinal axis of cells. When podocytes were treated with UTP or L-Hcy, the F-actin arrangement in podocytes was disturbed, and such fiber proteins were even markedly reduced. However, inhibition of Rac1 


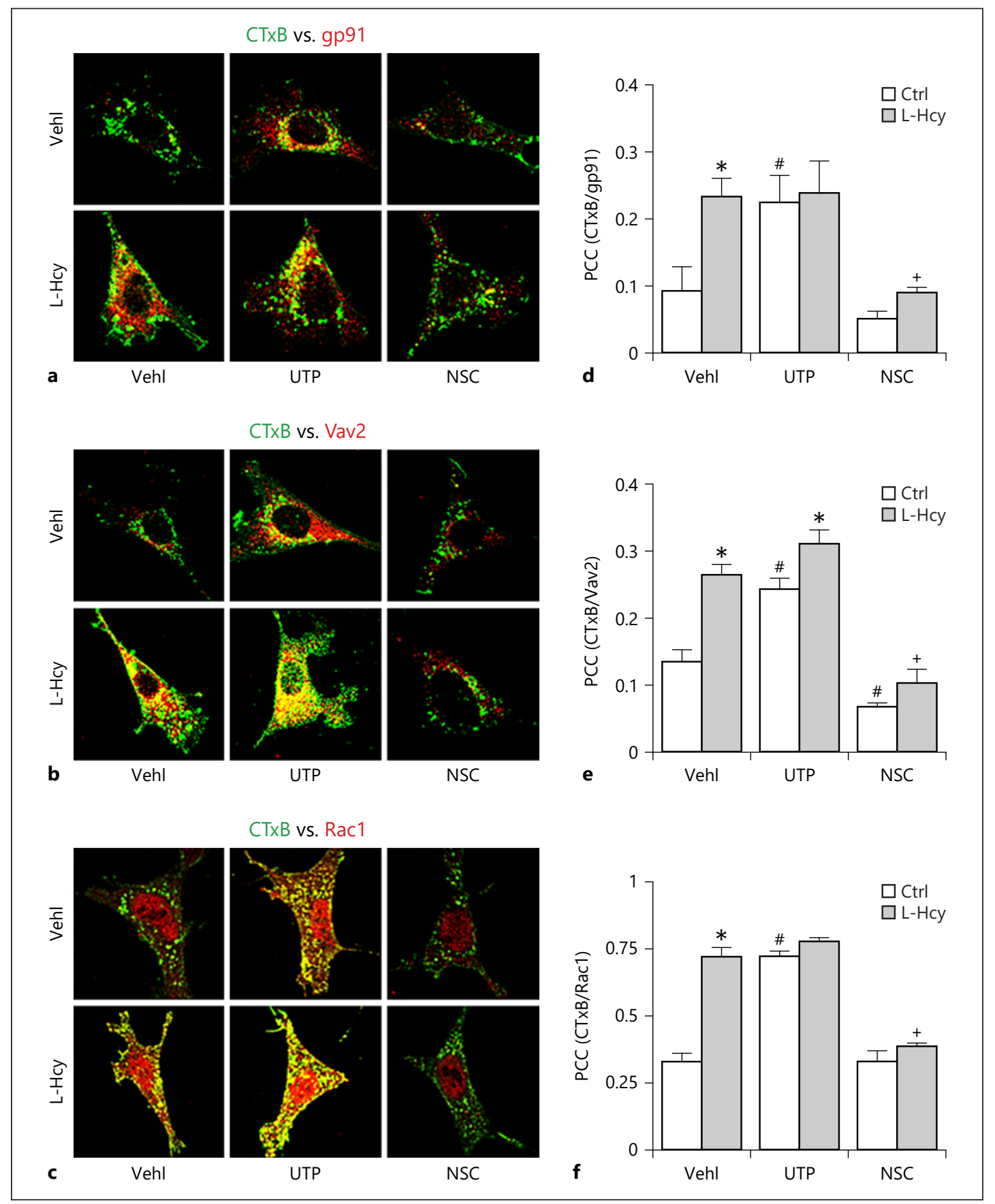

Fig. 4. L-Hcy and UTP treatment increased formation of LR clusters in podocytes. a-c Colocalization of CTxB (green) with gp91 (red), Vav2 (red) and CTxB (green) with Rac1 (Red) in cultured podocytes treated with UTP or NSC. $\mathbf{d - f}$ Summarized data showing the fold change of these colocalizations in correlation coefficient $(n=5) .{ }^{*} p<0.05$ versus Ctrl; ${ }^{\#} p<0.05$ versus Vehl/Ctrl; ${ }^{+} p<0.05$ versus Vehl/L-Hcy. UTP, uridine triphosphate; NSC, NSC-23766; Hcy, homocysteine.

activity by NSC23766 attenuated L-Hcy-induced decrease and rearrangement of F-actin (Fig. 3e). This F-actin fiber staining was analyzed by densitometry, and the data are presented in Figure 3f, clearly showing that L-Hcy led to derangement of F-actin expression and their structural arrangement. 


\section{Kidney \\ Blood Pressure \\ Research}

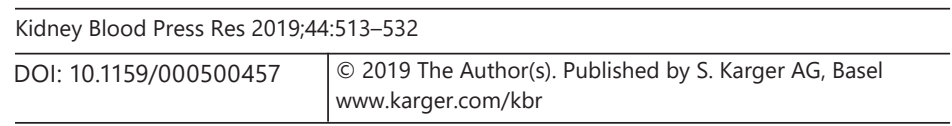

Zhang et al.: NLRP3 Inflammasome Activation via LR Redox Signaling Platforms

Fig. 5. Changes in L-Hcy-stimulated $\mathrm{O}_{2}^{-}$production in podocytes via NOX during Rac1 inhibition or activation. $\mathrm{O}_{2}^{-}$production $(n=5)$ in UTP- and NSC-treated podocytes in the presence or absence of Hcy. ${ }^{*} p<0.05$ versus Ctrl; ${ }^{\#} p<$ 0.05 versus Vehl/Ctrl; ${ }^{+} p<0.05$ versus Vehl/L-Hcy. Vehl, vehicle; UTP, uridine triphosphate; NSC, NSC-23766; Hcy, homocysteine; SOD, superoxide dismutase.

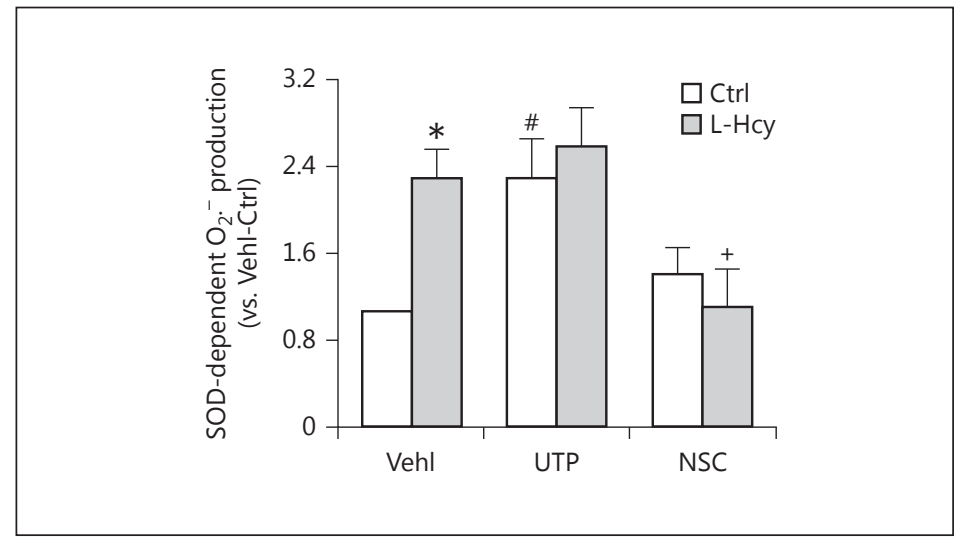

The Formation and Activation of LR Redox Signaling Platforms

To explore the mechanism by which L-Hcy activates NLRP3 inflammasomes, we determined the formation and activation of LR redox signaling platforms associated with NOX and its partners, Rac1. In vehicle-treated podocytes, there is no colocalization of LR marker CTxB with NOX subunit gp9 $1^{\text {phox }}$ as shown in confocal microscopic images (Fig. 4a). L-Hcy increased colocalization of CTxB with gp91 ${ }^{\text {phox }}$, Rac1, or Vav2, an activator of Rac1 in podocytes as shown by yellow patches in podocytes. Similarly, activation of Rac1 by UTP increased colocalization of CTxB with gp91 phox, Rac1, or Vav2, mimicking the effects of L-Hcy (Fig. 4a-c), suggesting increased formation of LR redox signaling platforms with Rac1/Vav2. Treatment of podocytes with NSC23766 had no marked effects on the colocalization of CTxB with gp91 ${ }^{\text {phox }}$, Rac1, or Vav2, but it blocked L-Hcy-increased colocalization of these signaling molecules. These results were summarized in Figure $4 \mathrm{~d}-\mathrm{f}$, showing that inhibition of Rac1 activity significantly blocked the formation of LR redox signaling platforms induced by L-Hcy and activation of Rac1 mimics L-Hcy-induced effects on this LR redox signaling platforms in podocytes.

We also examined the activation of LR redox signaling platforms in podocytes in response to different stimuli using ESR analysis. It was found that $\mathrm{O}_{2}{ }^{-}$production in podocytes was significantly increased by L-Hcy and UTP. Pretreatment of these cells with NSC23766 completely abolished L-Hcy-induced $\mathrm{O}_{2}^{-}$production, suggesting the importance of Rac1 activity to the activation of LR redox signaling platforms to produce $\mathrm{O}_{2}^{-}$in podocytes (Fig. 5).

Rac1 Inhibition Prevented hHcy-Induced NLRP3 Inflammasome Formation and Activation in Glomeruli

In in vivo animal experiments, we also tested whether Rac1-associated redox signaling platforms mediate hHcy-induced inflammasome formation and activation in podocytes and lead to glomerular injury. A mouse model with Nlrp3 gene deletion was used to determine whether the role of Rac1 in mediating Hcy-induced podocyte injury and glomerular sclerosis is dependent upon NLRP3 inflammasome activation. In both Nlrp3 gene KO and wild-type $\left(\mathrm{Nlrp3}^{+/+}\right)$mice, the FF diet for 6 weeks significantly increased plasma total Hcy levels $(38 \pm$ 5 vs. $13 \mu \mathrm{M}$ of control). Neither gene deletion nor treatments with UTP or NSC23766 altered plasma Hcy levels.

By confocal microscopy, we found that in hHcy mice and UTP-treated mice even fed the ND, there was increased colocalization of NLRP3 with ASC and NLRP3 with caspase-1 in glomeruli, which was mainly located in podocytes as shown in previous studies $[14,18,21]$. However, this colocalization of NLRP3 with ASC and NLRP3 with caspase-1 in glomeruli was 
Kidney

Blood Pressure

Research

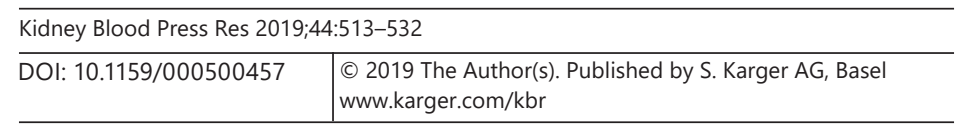

Zhang et al.: NLRP3 Inflammasome Activation via LR Redox Signaling Platforms

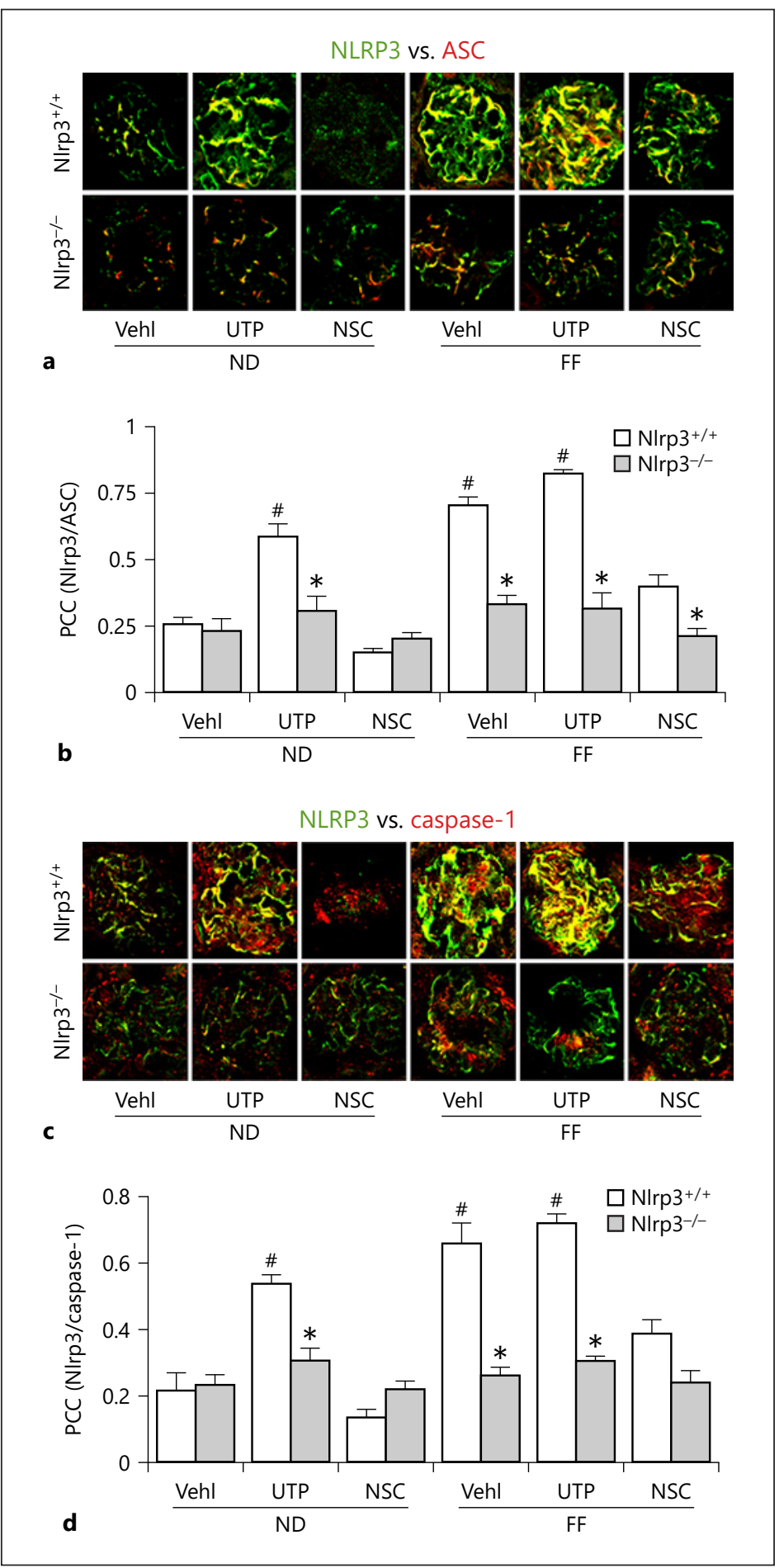

Fig. 6. HHcy and UTP administration enhanced glomerular NLRP3 inflammasome formation in the kidney of wild-type $\left(\mathrm{Nlrp3}^{+/+}\right)$ mice, but not in Nlrp3 knockout mice. Colocalization of NLRP3 (green) with ASC (red) or caspase-1 (red) in mouse glomeruli following UTP or NSC administration in FF- or ND-fed mice. Summarized data showing the fold changes in correlation coefficient for the colocalization of NLRP3 with ASC or with caspase-1 $(n=6)$. * $p<0.05$ versus $\mathrm{Nlrp3}^{+/+}$; \# $p<$ 0.05 versus Vehl/ND/Nlrp3 $3^{+/+}$. Vehl, vehicle; UTP, uridine triphosphate; NSC, NSC-23766; Nlrp3 $^{+/+,}$WT/WT; Nlrp3-/-, Nlrp3 KO; ND, normal diet; FF, folatefree diet; ASC, apoptosis-associated speck-like protein. 


\section{Kidney \\ Blood Pressure \\ Research}

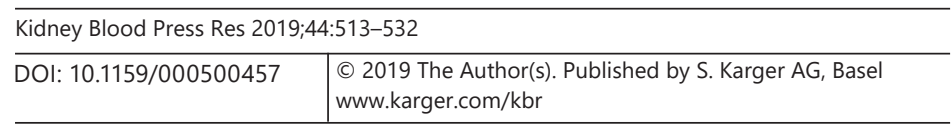

Zhang et al.: NLRP3 Inflammasome Activation via LR Redox Signaling Platforms

Fig. 7. In vivo administration of Rac1 inhibitor, NSC23766, or Nlrp3 gene deletion blocked glomerular NLRP3 inflammasome activation during hHcy in mice. Representative images of immunohistochemical staining of IL-1 $\beta$ both in $\mathrm{Nlrp}^{+/+}$and in Nlrp3 KO glomerular podocytes in mice on the normal or FF diet with UTP and NSC treatment. Summarized data depicting relative intensity of IL-1 $\beta$ brown staining $(n=6)$. * $p<0.05$ versus Nlrp3 ${ }^{+/+}$; \# $p<$ 0.05 versus Vehl/ND/ Nlrp3 ${ }^{+/+}$. Vehl, vehicle; UTP, uridine triphosphate; NSC, NSC-23766; $\mathrm{Nlrp}^{+/+}$, WT/WT; Nlrp3 ${ }^{-/-}$, Nlrp3 KO; ND, normal diet; FF, folatefree diet; IL-1 $\beta$, interleukin-1 $\beta$.

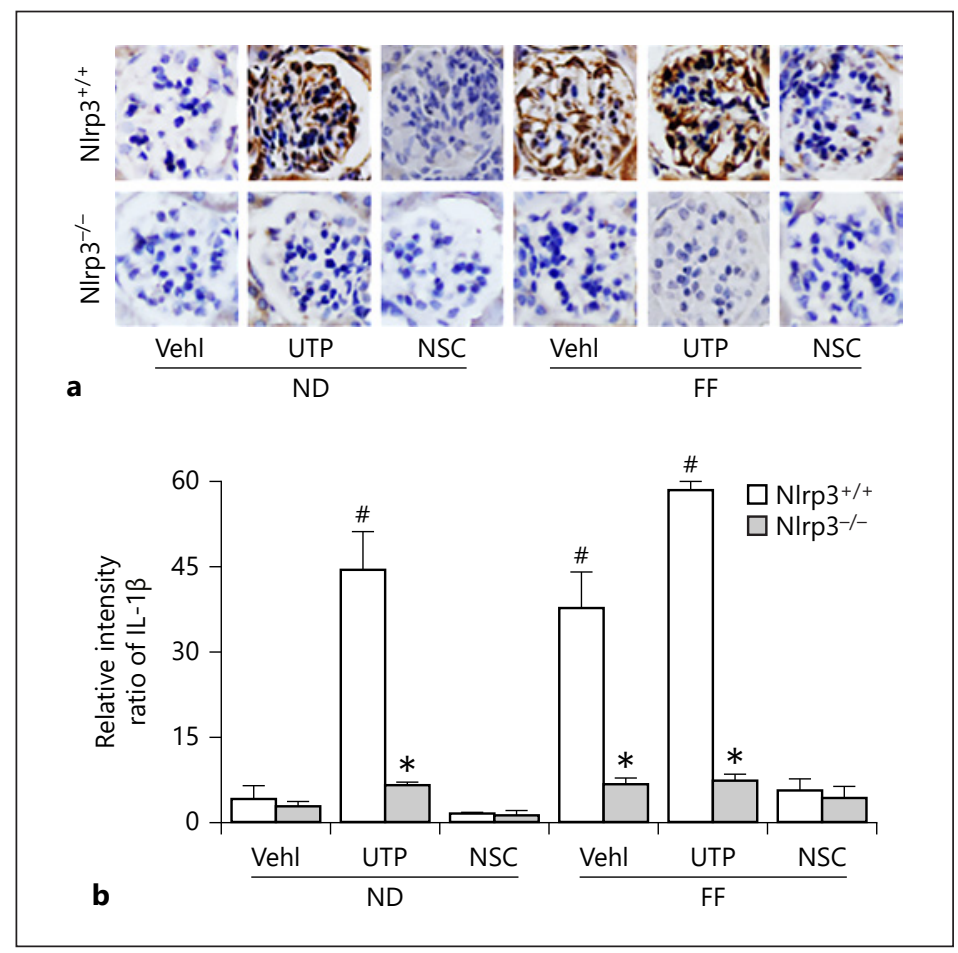

inflammasome formation in glomeruli induced by hHcy. These inhibitory effects of NSC23766 were similar to that observed in mice with Nlrp3 gene deletion, suggesting that Rac1 inhibition indeed suppresses hHcy-induced glomerular injury through its action on NLRP3 inflammasomes.

Since activation of the NLRP3 inflammasome is associated with the maturation of proinflammatory cytokine, IL-1 $\beta$, we measured the IL-1 $\beta$ levels in mouse glomeruli using immunohistochemistry. There was a remarkable increase in IL-1 $\beta$ levels in glomeruli of $\mathrm{Nlrp3}^{+/+}$mice on the FF diet with and without UTP treatment. UTP treatment even increased the IL-1 $\beta$ level in glomeruli of mice on the ND. However, knockout of Nlrp3 abolished glomerular IL-1 $\beta$ increases in all treatment groups of mice (Fig. 7a). Quantitation of IL-1 $\beta$ staining showed that hHcy and UTP significantly increased glomerular IL-1 $\beta$ level, indicating local NLRP3 inflammasome activation. This NLRP3 inflammasome activation in hHcy was blocked in Nlrp3 gene KO mice and in mice with treatment of Rac1 inhibitor, NSC23766 (Fig. 7b).

Involvement of Rac1 Activity in hHcy-Induced Podocyte Injury and Glomerular Sclerosis in Mice

To determine whether hHcy leads to podocyte injury and consequent glomerular sclerosis, we examined changes in podocyte functional and injury markers in mouse glomeruli and corresponding alterations of glomerular function. It was demonstrated that in both hHcy $\mathrm{Nlrp}^{+/+}$mice and UTP-treated mice, there were dramatic decreases in podocin staining and increases in desmin staining. Rac1 inhibition by NSC23766 administration resulted in amelioration of hHcy-induced podocyte injury as shown by restoration of podocin expression levels. Moreover, UTP-treated $\mathrm{Nlrp3}^{+/+}$mice exhibited a high abundance of desmin expression in glomeruli of both ND- and FF-fed groups, whereas NSC23766 administration reduced hHcyinduced increases in glomerular desmin staining. In Nlrp3 gene KO mice, however, hHcy and 
Kidney

Blood Pressure

Research

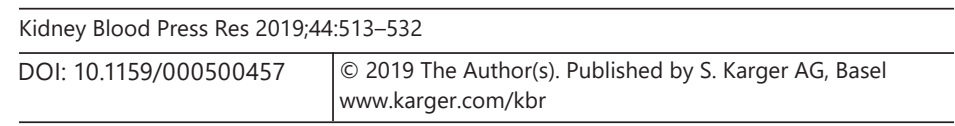

Zhang et al.: NLRP3 Inflammasome Activation via LR Redox Signaling Platforms
Fig. 8. Rac1 inhibitor, NSC23766, or Nlrp3 gene deletion protected glomerular podocytes from injury induced by hHcy in mice. Immunofluorescent staining of podocyte-specific markers, podocin (a) and desmin (c), in mouse glomeruli $(n=6)$. Quantification of relative protein intensity in glomeruli was shown in the right lane. ${ }^{*} p<0.05$ versus Nlrp3 $3^{+/}$; \# $p<0.05$ versus Vehl/ND/ $\mathrm{Nlrp}^{+/+}$. Vehl, vehicle; UTP, uridine triphosphate; NSC, NSC-23766; $\mathrm{Nlrp3}^{+/+}$, WT/WT; Nlrp3-/-, Nlrp3 KO, ND, normal diet, FF, folate-free diet.

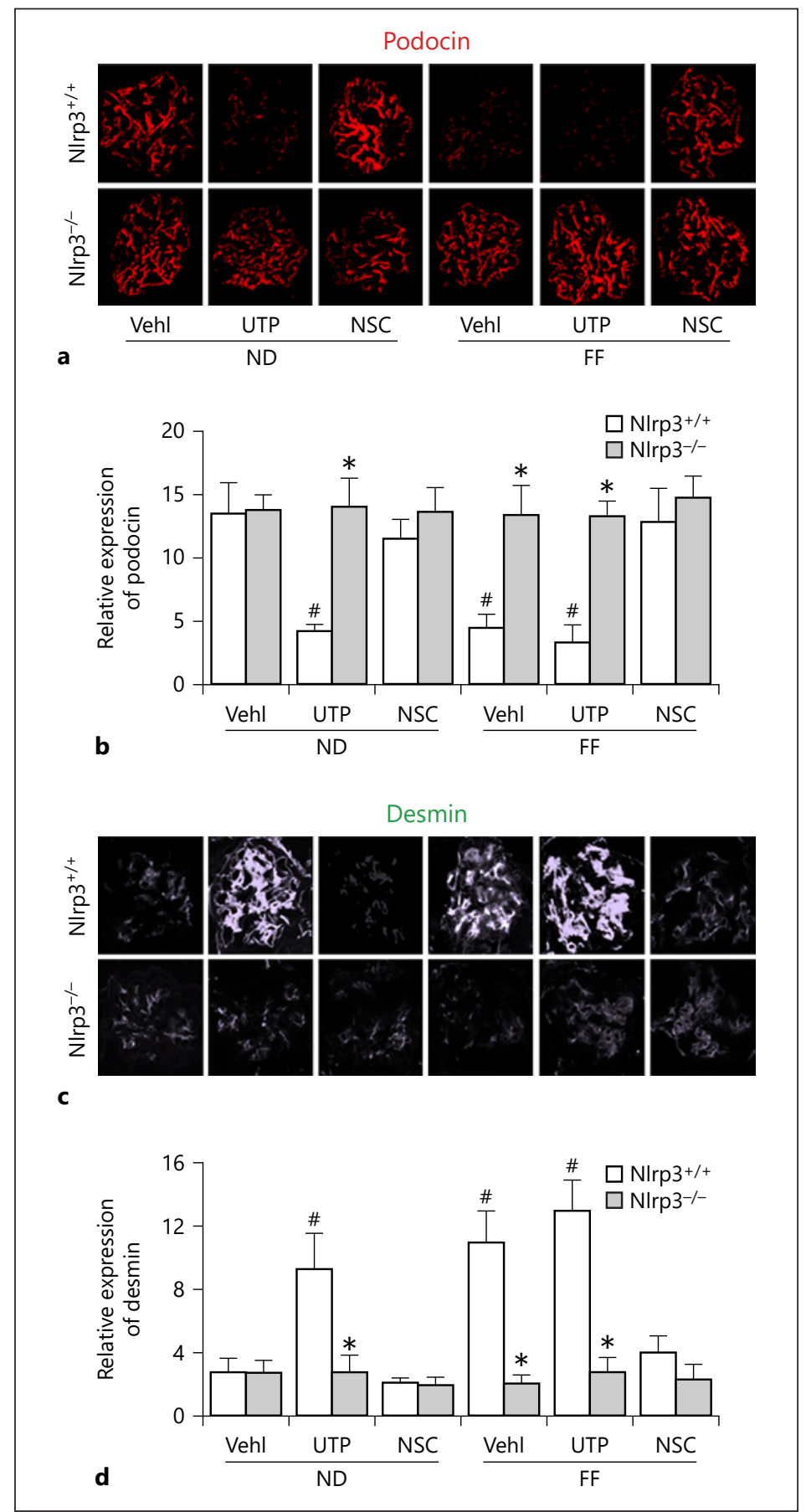

UTP had no effects on podocyte protein level (Fig. 8a, c). The summarized data are presented in Figure $8 \mathrm{~b}$ and d, showing that podocyte injury was significantly increased in mice with hHcy and mice with Rac1 activator. Podocyte injury in mice with hHcy was significantly attenuated by inhibition of Rac1 activity.

As illustrated in Figure 9a, there was obvious proteinuria in $\mathrm{Nlrp}^{+/+}$mice with hHcy or treated with UTP. This proteinuria induced by hHcy was significantly attenuated by NSC23766 administration. However, proteinuria was not observed in Nlrp3 gene KO mice 


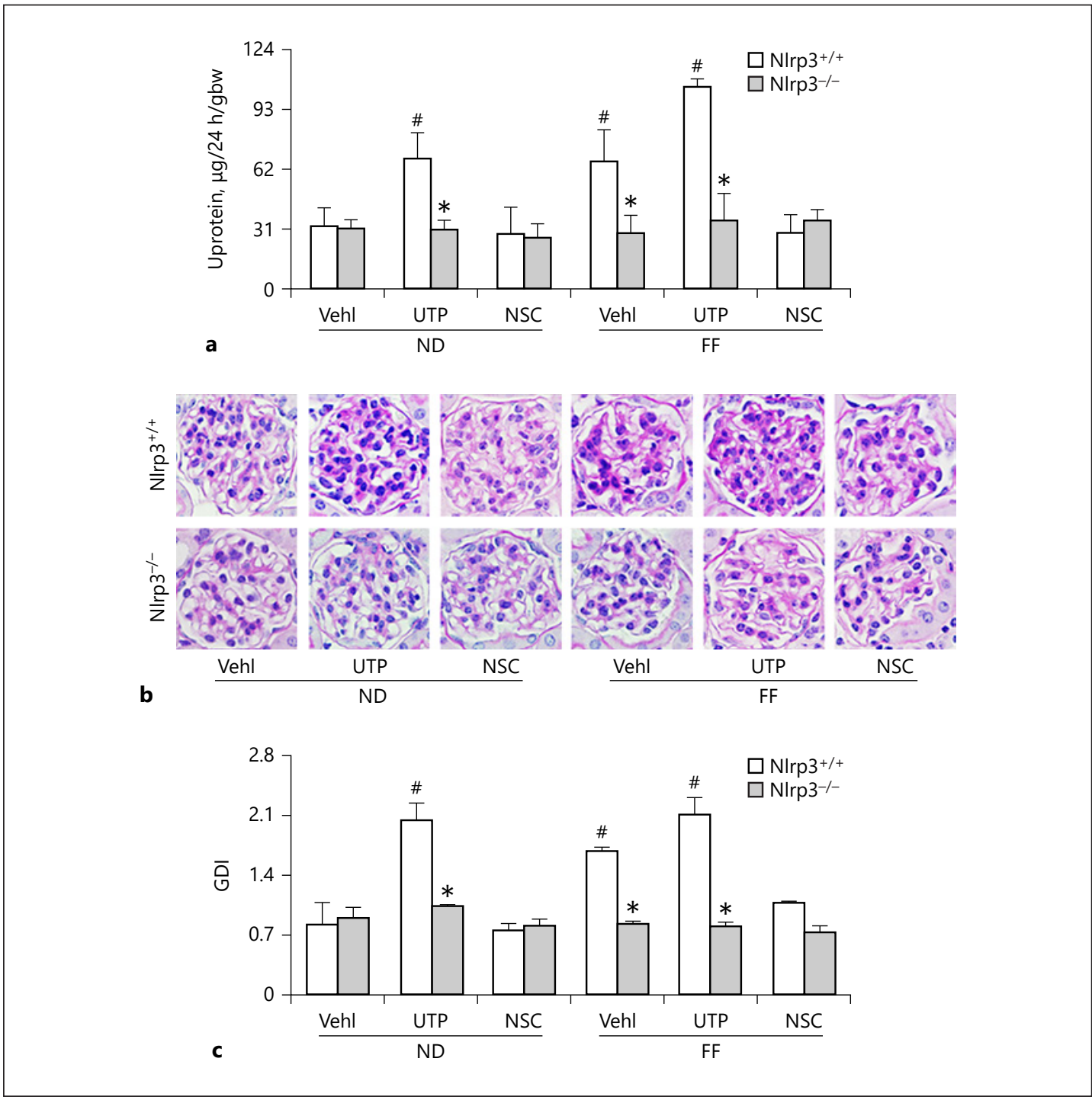

Fig. 9. In vivo inhibition of Rac1 activity or Nlrp3 gene deletion abolished hHcy-induced glomerular damage in mice. a Urinary protein $(n=4)$ measurements in 24-h urine samples of hHcy mice receiving FF, UTP, and NSC treatment. b Microscopic microphotograph of glomerular structure in periodic acid-Schiff-stained kidney sections. c Semiquantitative assessment of GDI from periodic acid-Schiff images $(n=6){ }^{*} p<0.05$ versus $\mathrm{Nlrp3}^{+/+}$; ${ }^{*} p<0.05$ versus Vehl/ND/ Nlrp3 ${ }^{+/+}$. UTP, uridine triphosphate; NSC, NSC-23766; Nlrp3 $3^{+/+}$, WT/ WT; Nlrp3 ${ }^{-/-}$, Nlrp3 KO; ND, normal diet, FF, folate-free diet; U, urinary; GDI, glomerular damage index.

no matter what treatments were given or not. Morphological examinations showed that hHcy and UTP administration resulted in glomerular sclerosis in $\mathrm{Nlrp3}^{+/+}$mice, but not in Nlrp3 KO mice. When mice received NSC23766, hHcy-induced glomerular injury was almost completely blocked, which was similar to that observed in Nlrp3 KO mice (Fig. 9b). Calculated GDI was summarized in Figure 9c. It was demonstrated that UTP in $\mathrm{Nlrp3}^{+/+}$mice even on the ND and hHcy mice with and without UTP treatment exhibited significantly increased glomerular damage. Inhibition of Rac1 activity by administration of NSC23766 almost completely blocked increases in GDI in mice with hHcy, which was similar to that observed in Nlrp3 KO mice. 


\section{Kidney \\ Blood Pressure \\ Research}

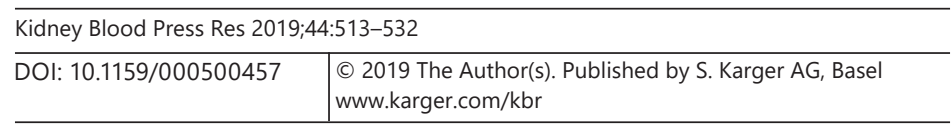

Zhang et al.: NLRP3 Inflammasome Activation via LR Redox Signaling Platforms

\section{Discussion}

The major goal of the present study was to determine the contribution of Rac1 and its association with LR clustering to NLRP3 inflammasome activation and podocyte injury induced by hHcy and to test whether this NLRP3 inflammasome activation can serve as a therapeutic target for prevention or treatment of hHcy-induced glomerular sclerosis. Our results demonstrated that inhibition of the Rac1 activity attenuated NLRP3 inflammasome formation, decreased caspase- 1 activation, and subsequently reduced IL- $1 \beta$ production in L-Hcy-treated podocytes. Conversely, activation of Rac1 enhanced NLRP3 inflammasome formation and activation in these cells independent of L-Hcy treatment. These effects of altered Rac1 activity were found to be associated with activation of LR redox signaling platforms to produce ROS. Additionally, we showed that administration of Rac1 inhibitor, NSC23766, in vivo attenuated hHcy-induced inflammasome activation and glomerular dysfunction, highlighting the significance of pharmacologically targeting Rac1 activity and associated LR redox signaling platforms.

Recent studies have indicated that the activation of inflammasomes in most of the phagocytes or different non-phagocytes serves as an intracellular machinery to trigger inflammatory response in a variety of tissues or organs [22]. So far, several inflammasomes have been found, such as NLRP3, NLRP1, NLRC4, and RIG-I inflammasomes [23]. Among these inflammasomes, however, NLRP3 inflammasome is widely studied and shown to occur in multiple mammalian cells such as endothelial cells, epithelial cells, neurons, podocytes, cardiac muscle cells, or macrophages and importantly participates in the development of chronic degenerative diseases such as atherosclerotic diseases, Alzheimer's disease, lung fibrosis, and end-stage renal disease [15, 19, 24]. The activation of NLRP3 inflammasomes promotes the maturation and secretion of proinflammatory cytokines such as IL-1 $\beta$, IL-18, HMGB1, or other products, which instigate the tissue inflammatory response or directly alter cellular metabolism or cell function to produce pathogenic changes via their uncanonical effects $[14,19,24]$. In our previous studies, we revealed that the NLRP3 inflammasome is activated during hHcy to produce podocyte injury leading to chronic glomerular inflammation and sclerosis. This NLRP3 inflammasome activation is associated with NOX activation and redox signaling during hHcy $[5,9,18]$. However, how this NOX-mediated redox signaling is activated and whether this redox signaling is critically involved in podocyte NLRP3 activation and glomerular injury remain to be determined.

To answer these questions, the present study first tested whether Rac1, a GTPase that controls NOX activation, participates in Hcy-induced NLRP3 inflammasome formation and activation. It was shown that in cultured podocytes, Rac1 activator mimicked L-Hcy-induced NLRP3 inflammasome formation and activation, while Rac1 inhibition blocked them. Corresponding to the NLRP3 inflammation activation or inhibition, enhanced Rac1 activity produced podocyte injury, while its inhibition protected podocytes from L-Hcy-induced injury. These results indicate that Rac1 plays a critical role in triggering or regulating NLRP3 inflammasomes in podocytes and thereby may contribute to the development of podocyte dysfunction and consequent glomerular injury. In this regard, a recent study has demonstrated that Hcy-induced podocyte dysfunction and injury is dependent on the activation of Vav2, a guanine nucleotide exchange factor family of oncogenes that regulates Rac1 activity, which is also associated with its action on NLRP3 inflammasome. It was suggested that activation of Vav2 in podocytes might enhance Rac1 activity, thereby promoting the aggregation of the multi-protein NOX complex to produce $\mathrm{O}_{2}{ }^{-}$and thus result in NLRP3 inflammasome activation and initiation of glomerular injury. This $\mathrm{O}_{2}{ }^{-}$or ROS-mediated activation of NLRP3 inflammasome has also been reported in different types of cells including podocytes, which is now commonly considered as a redox regulatory pathway of the NLRP3 inflammasome. It 


\section{Kidney \\ Blood Pressure \\ Research}

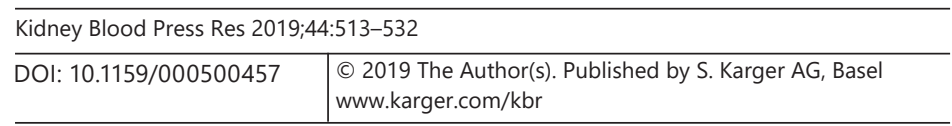

Zhang et al.: NLRP3 Inflammasome Activation via LR Redox Signaling Platforms

has been proposed that the role of ROS in the regulation of NLRP3 inflammasomes is via thioredoxin interacting proteins, which serve as a binding partner to NLRP3. Their association or binding is a key mechanism responsible for inflammasome activation. ROS may produce more thioredoxin interacting protein binding to NLRP3 leading to inflammasome formation and activation due to antioxidant thioredoxin dissociation [15]. In other studies on different tissues or cells, Rac1 was also found to be an activator of NLRP3 inflammasome, which may trigger the inflammatory response to different pathological challenges [25, 26]. All the results confirm the idea that Rac1 is a critical regulatory molecule for NLRP3 inflammasome activation to trigger tissue inflammatory response. In the kidney, this Rac1-mediated regulation occurs in podocytes, which may importantly maintain the structural and functional integrity of podocyte and glomeruli.

Next, we addressed how activated NOX and ROS production due to L-Hcy-enhanced Rac1 activity induces NLRP3 inflammasome activation. In previous studies, it was demonstrated that Hcys-induced podocyte injury is attributed to the clustering of LRs with recruitment and assembling of NOX subunits to produce $\mathrm{O}_{2}^{-}$[18]. This led us to hypothesize that Rac1 activation may cause LR clustering to form NOX-mediated redox signaling platforms and thereby contributes to the formation and activation of NLRP3 inflammasomes. By confocal microscopy, we indeed confirmed that L-Hcy increased colocalization of CTxB with gp91 ${ }^{\text {phox }}$, Rac1, or Vav2 in podocytes, which was mimicked by the activation of Rac1 with UTP. The results suggest that the LR redox signaling platforms are formed with Rac1/Vav2. However, inhibition of Rac1 activity significantly blocked the formation of LR redox signaling platforms induced by $\mathrm{L}-\mathrm{Hcy}$. Corresponding to the NOX-associated LR clustering, $\mathrm{O}_{2}^{-}$production significantly increased by L-Hcy and UTP, but blocked by Rac1 inhibitor, NSC23766. It is clear that Rac1 activity participates in the effects of L-Hcy to stimulate activation of LR redox signaling platforms and consequent $\mathrm{O}_{2}^{-}$in podocytes. These results were beyond our original thought that LR clustering may aggregate NOX subunits with Rac1 and Vav2 to activate redox signaling by production of $\mathrm{O}_{2}^{-}$because activation of Rac1 can even stimulate LR clustering to form NOXcentered signaling platforms. This suggests that Rac1 not only serves as a cofactor for the NOX activity during hHcy but also is an activator of LR redox signaling platforms. The present study did not attempt to examine why Rac1 activation or inhibition alters LR clustering, which will be an interesting project for us to pursue further. In previous studies on other cell types, however, LR clustering or associated protein trafficking or aggregation was indeed found to be Rac1 dependent. It has been shown that the binding of Rac1 to some protein domain such as phosphoinositide 3-kinase isoforms, p110 3 , promoted LR localization of this protein, suggesting that Rac1 binding is important for the PI2K signaling platforms on the cell membrane [27]. In another study on dentritic cell development from human stem cells, Rac1 was found to be essential and its activation results in rapid recruitment into LRs, which controls the transport of essential immunostimulatory molecules to the dentritic cell surface. These studies suggest that LR signaling platforms for immunoregulatory signal transduction rely on Rac1 activation [28]. In addition, the level and size distribution pattern of LRs are found to importantly participate in raft-associated stress sensing and signaling. The functionality of Rac1, in particular, activation or palmitoylation alters its relocalization to the surface membrane, thereby resulting in membrane hyperfluidization-induced remodeling of membrane microdomains, namely, LRs. This LR remodeling including platform formation or deformation is controlled by Rac1 activity [29].

It should be noted that Rac1 GTPase may mediate hHcys-induced NLRP3 inflammasome activation not only through redox signaling mechanism. For example, Rac1 activation associated with Vav activity has been reported to be fibrotic or sclerotic, which may be induced via the adaptor molecule Cbl-b [30,31]. On the other hand, there were reported that deficiency of Rac1 activity induced by Vav2 knockout mice produced collagen accumulation in 


\section{Kidney \\ Blood Pressure \\ Research}

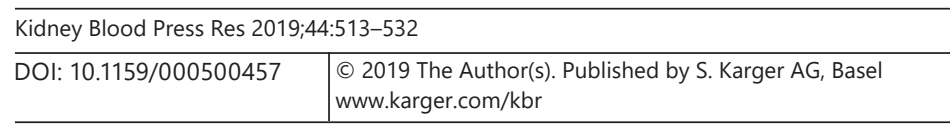

Zhang et al.: NLRP3 Inflammasome Activation via LR Redox Signaling Platforms

different organs including kidneys, which may be due to generous injurious pathology given that other signaling pathways may be malfunctioning such as a chronic stimulation of the renin/angiotensin II and sympathetic nervous systems, as they proposed [32]. In epithelial cells, NLRP3 inflammasome activation was shown to require the formation of the focal adhesion complex including focal adhesion kinase, Rac1, and other components, which may not necessarily link to redox signaling [33]. Therefore, we should be cautious in explaining the protective effects of NSC, which may be limited to hHcys-induced glomerular injury.

To address the functional relevance of Rac1-mediated LR redox signaling activation, we performed animal experiments to observe the effects of activation or inhibition of Rac1 on podocyte NLRP3 inflammasome activation and glomerular injury in mice. It was found that activation of Rac1 resulted in significant enhancement of the NLRP3 inflammasome formation and activation in podocytes of mouse glomeruli, which was similar to that observed in mice with hHcy. This enhanced inflammasome formation and activation in podocytes were accompanied by podocyte injury as shown by increased expression of podocyte injury factor desmin and reduction of podocyte functional protein podocin with consequence of proteinuria and glomerular sclerosis. However, these effects of Rac1 activation or hHcy to activate NLRP3 inflammasome and glomerular injury in vivo could not be observed in Nlrp3 gene knockout mice, suggesting that it is the NLRP3 inflammasome that is the target of Rac1 activation and $\mathrm{hHcy}$. This view was supported by the results obtained by administration of Rac1 inhibitor to mice, where hHcy-induced inflammasome activation and podocyte injury as well as glomerular dysfunction or sclerosis were completely blocked. These results from animal studies together suggest that Rac1-mediated NLRP3 inflammasome activation is an important mechanism mediating podocyte injury and glomerular sclerosis, which may mediate the pathological changes in glomeruli of mice with hHcy. Since NLRP3 inflammasome activation has been reported to contribute to the development of different chronic glomerular diseases leading to chronic renal insufficiency such as chronic glomerulonephritis, hyperhomocysteinemic nephropathy, diabetic nephropathy, and glomerular injury or sclerosis in obesity [14, 34-37], the results from the present study may suggest that the pathogenic role of Rac1-mediated NLRP3 inflammasome activation may be a common mechanism for these chronic glomerular diseases. With respect to the mechanisms by which NLRP3 inflammasome activation produces glomerular injury, there are reports that the inflammatory response was indeed enhanced in glomeruli during hHcys when this inflammasome was activated. This glomerular inflammatory response was characterized by recruitment of macrophages and T-cells. In addition, there is evidence that suppression of inflammasome activation also protected the functional and structural integrity of podocytes, even before a typical inflammatory response is instigated. This noncanonical, noninflammatory, or direct effect on podocytes has been reported to be associated with IL-1 $\beta$-induced podocyte dysfunction. Therefore, in addition to the inflammatory response, the early effect of activated inflammasome products, such as IL-1 $\beta$, directly on podocytes also importantly contributes to podocyte injury [14]. Taken together, it is obvious that targeting Rac1-mediated NLRP3 inflammasome activation may be a novel therapeutic strategy for the prevention and treatment of various glomerular diseases associated with chronic inflammation and direct podocyte injury in glomeruli.

In summary, the present study demonstrated that pharmacologically changed Rac1 activity altered NLRP3 inflammasome formation and activation in podocytes and corresponding podocyte and glomerular function. Inhibition of Rac1 activity blocked the effects of elevated Hcy on NLRP3 inflammasome activation and related podocyte and glomerular pathology. It was also shown that Rac1 action on NLRP3 inflammation activation and podocyte function was associated with LR raft redox signaling. Based on these results, we concluded that Rac1 inhibition may suppress NLRP3 inflammasome activation in podocytes and thereby exerts its beneficial action of podocyte injury and glomerular sclerosis during hHcys. 
Kidney
Blood Pressure
Research

\begin{tabular}{l|l}
\hline Kidney Blood Press Res 2019;44:513-532 \\
\hline DOI: 10.1159/000500457 & $\begin{array}{l}\text { ○ 2019 The Author(s). Published by S. Karger AG, Basel } \\
\text { www.karger.com/kbr }\end{array}$ \\
\hline
\end{tabular}

Zhang et al.: NLRP3 Inflammasome Activation via LR Redox Signaling Platforms

\section{Acknowledgment}

This study was supported by grants from the National Institutes of Health (DK054927, DK120491, HL057244, and HL075316).

\section{Disclosure Statement}

The authors declare no conflicts of interest.

\section{References}

1 Ducloux D, Motte G, Challier B, Gibey R, Chalopin JM. Serum total homocysteine and cardiovascular disease occurrence in chronic, stable renal transplant recipients: a prospective study. J Am Soc Nephrol. 2000 Jan; 11(1):134-7.

2 Moustapha A, Gupta A, Robinson K, Arheart K, Jacobsen DW, Schreiber MJ, et al. Prevalence and determinants of hyperhomocysteinemia in hemodialysis and peritoneal dialysis. Kidney Int. 1999 Apr;55(4):1470-5.

3 Robinson K, Gupta A, Dennis V, Arheart K, Chaudhary D, Green R, et al. Hyperhomocysteinemia confers an independent increased risk of atherosclerosis in end-stage renal disease and is closely linked to plasma folate and pyridoxine concentrations. Circulation. 1996 Dec;94(11):2743-8.

4 Conley SM, Abais-Battad JM, Yuan X, Zhang Q, Boini KM, Li PL. Contribution of guanine nucleotide exchange factor Vav2 to NLRP3 inflammasome activation in mouse podocytes during hyperhomocysteinemia. Free Radic Biol Med. 2017 May;106:236-44.

5 Xia M, Conley SM, Li G, Li PL, Boini KM. Inhibition of hyperhomocysteinemia-induced inflammasome activation and glomerular sclerosis by NLRP3 gene deletion. Cell Physiol Biochem. 2014;34(3):829-41.

6 Martinon F, Mayor A, Tschopp J. The inflammasomes: guardians of the body. Annu Rev Immunol. 2009;27(1): 229-65.

7 Lamkanfi M, Dixit VM. Inflammasomes and their roles in health and disease. Annu Rev Cell Dev Biol. 2012; 28(1):137-61.

8 Latz E, Xiao TS, Stutz A. Activation and regulation of the inflammasomes. Nat Rev Immunol. 2013 Jun;13(6): 397-411.

9 Yi F, Zhang AY, Li N, Muh RW, Fillet M, Renert AF, et al. Inhibition of ceramide-redox signaling pathway blocks glomerular injury in hyperhomocysteinemic rats. Kidney Int. 2006 Jul;70(1):88-96.

10 Gill PS, Wilcox CS. NADPH oxidases in the kidney. Antioxid Redox Signal. 2006 Sep-Oct;8(9-10):1597-607.

11 Simons K, Ikonen E. Functional rafts in cell membranes. Nature. 1997 Jun;387(6633):569-72.

12 Simons K, Toomre D. Lipid rafts and signal transduction. Nat Rev Mol Cell Biol. 2000 Oct;1(1):31-9.

13 Wan C, Su H, Zhang C. Role of NADPH Oxidase in Metabolic Disease-Related Renal Injury: an Update. Oxid Med Cell Longev. 2016;2016:7813072.

14 Abais JM, Zhang C, Xia M, Liu Q, Gehr TW, Boini KM, et al. NADPH oxidase-mediated triggering of inflammasome activation in mouse podocytes and glomeruli during hyperhomocysteinemia. Antioxid Redox Signal. 2013 May;18(13):1537-48.

15 Abais JM, Xia M, Li G, Chen Y, Conley SM, Gehr TW, et al. Nod-like receptor protein 3 (NLRP3) inflammasome activation and podocyte injury via thioredoxin-interacting protein (TXNIP) during hyperhomocysteinemia. ] Biol Chem. 2014 Sep;289(39):27159-68.

16 Yao HY, Chen L, Xu C, Wang J, Chen J, Xie QM, et al. Inhibition of Rac activity alleviates lipopolysaccharideinduced acute pulmonary injury in mice. Biochim Biophys Acta. 2011 Jul;1810(7):666-74.

17 Boini KM, Xia M, Abais JM, Xu M, Li CX, Li PL. Acid sphingomyelinase gene knockout ameliorates hyperhomocysteinemic glomerular injury in mice lacking cystathionine- $\beta$-synthase. PLoS One. 2012;7(9):e45020.

18 Zhang C, Hu JJ, Xia M, Boini KM, Brimson C, Li PL. Redox signaling via lipid raft clustering in homocysteineinduced injury of podocytes. Biochim Biophys Acta. 2010 Apr;1803(4):482-91.

19 Abais JM, Xia M, Li G, Gehr TW, Boini KM, Li PL. Contribution of endogenously produced reactive oxygen species to the activation of podocyte NLRP3 inflammasomes in hyperhomocysteinemia. Free Radic Biol Med. 2014 Feb;67:211-20.

20 Raij L, Azar S, Keane W. Mesangial immune injury, hypertension, and progressive glomerular damage in Dahl rats. Kidney Int. 1984 Aug;26(2):137-43.

21 Youm YH, Adijiang A, Vandanmagsar B, Burk D, Ravussin A, Dixit VD. Elimination of the NLRP3-ASC inflammasome protects against chronic obesity-induced pancreatic damage. Endocrinology. 2011 Nov; 152(11): 4039-45.

22 Schroder K, Tschopp J. The inflammasomes. Cell. 2010 Mar;140(6):821-32.

23 Lupfer C, Malik A, Kanneganti TD. Inflammasome control of viral infection. Curr Opin Virol. 2015 Jun;12: $38-46$. 
24 Zhang Y, Li X, Pitzer AL, Chen Y, Wang L, Li PL. Coronary endothelial dysfunction induced by nucleotide oligomerization domain-like receptor protein with pyrin domain containing 3 inflammasome activation during hypercholesterolemia: beyond inflammation. Antioxid Redox Signal. 2015 May;22(13):1084-96.

25 Hummler JK, Dapaah-Siakwan F, Vaidya R, Zambrano R, Luo S, Chen S, et al. Inhibition of Rac1 Signaling Downregulates Inflammasome Activation and Attenuates Lung Injury in Neonatal Rats Exposed to Hyperoxia. Neonatology. 2017;111(3):280-8.

26 Eitel J, Meixenberger K, van Laak C, Orlovski C, Hocke A, Schmeck B, et al. Rac1 regulates the NLRP3 inflammasome which mediates IL-1beta production in Chlamydophila pneumoniae infected human mononuclear cells. PLoS One. 2012;7(1):e30379.

27 Cizmecioglu O, Ni J, Xie S, Zhao JJ, Roberts TM. Rac1-mediated membrane raft localization of PI3K/p110 $\beta$ is required for its activation by GPCRs or PTEN loss. eLife. 2016 Oct; 5:5.

28 Jaksits S, Bauer W, Kriehuber E, Zeyda M, Stulnig TM, Stingl G, et al. Lipid raft-associated GTPase signaling controls morphology and CD8+ T cell stimulatory capacity of human dendritic cells. J Immunol. 2004 Aug; 173(3):1628-39.

29 Gungor B, Gombos I, Crul T, Ayaydin F, Szabó L, Török Z, et al. Rac1 participates in thermally induced alterations of the cytoskeleton, cell morphology and lipid rafts, and regulates the expression of heat shock proteins in B16F10 melanoma cells. PLoS One. 2014 Feb;9(2):e89136.

30 Chen X, Abair TD, Ibanez MR, Su Y, Frey MR, Dise RS, et al. Integrin alpha1beta1 controls reactive oxygen species synthesis by negatively regulating epidermal growth factor receptor-mediated Rac activation. Mol Cell Biol. 2007 May;27(9):3313-26.

31 Chiang YJ, Kole HK, Brown K, Naramura M, Fukuhara S, Hu RJ, et al. Cbl-b regulates the CD28 dependence of T-cell activation. Nature. 2000 Jan;403(6766):216-20.

32 Sauzeau V, Jerkic M, López-Novoa JM, Bustelo XR. Loss of Vav2 proto-oncogene causes tachycardia and cardiovascular disease in mice. Mol Biol Cell. 2007 Mar;18(3):943-52.

33 Thinwa J, Segovia JA, Bose S, Dube PH. Integrin-mediated first signal for inflammasome activation in intestinal epithelial cells. J Immunol. 2014 Aug;193(3):1373-82.

34 Conley SM, Abais JM, Boini KM, Li PL. Inflammasome Activation in Chronic Glomerular Diseases. Curr Drug Targets. 2017;18(9):1019-29.

35 Abais JM, Xia M, Zhang Y, Boini KM, Li PL. Redox regulation of NLRP3 inflammasomes: ROS as trigger or effector? Antioxid Redox Signal. 2015 May;22(13):1111-29.

36 Zhang C, Boini KM, Xia M, Abais JM, Li X, Liu Q, et al. Activation of Nod-like receptor protein 3 inflammasomes turns on podocyte injury and glomerular sclerosis in hyperhomocysteinemia. Hypertension. 2012 Jul;60(1): 154-62.

37 Boini KM, Xia M, Abais JM, Li G, Pitzer AL, Gehr TW, et al. Activation of inflammasomes in podocyte injury of mice on the high fat diet: effects of ASC gene deletion and silencing. Biochim Biophys Acta. 2014 May; 1843(5): 836-45. 